Careful considerations in end-of-life care

Nursing and Residential Care (2015) vol. 17, (6)

\author{
Tushna Vandrevala ${ }^{1}$, Kritika Samsi ${ }^{2} \&$ Jill Manthorpe ${ }^{2}$ \\ ${ }^{1}$ Department of Psychology, Kingston University \\ ${ }^{2}$ Social Care Workforce Research Unit, King's College London
}

\begin{abstract}
With many older people expected to reach end of life in a residential home, it is important to consider the ramifications for that person, their family and friends and the staff who care for them; support must be available to all those involved.
\end{abstract}

With life expectancy in the UK increasing, many older people are likely to live in a care home in the later stages of their lives. In England 20\% of deaths occur in a care home (Office of National Statistics, 2012), and this figure is likely to be higher for people aged 75 and over (National end of life care Intelligence Network, 2010). Contrary to some views, the proportion of 'deaths in the usual place of residence' (DIUPR)—deaths in own home or a care home—is increasing, and consequently the proportion of deaths in hospital has fallen. This may be for a number of reasons, such as growing emphasis on people having a choice of where they die and a large proportion of people choosing this to be where they normally live. The consequence is that in many cases, care homes are the places where people die.

What do people mean by end-of-life care? 
Putting it simply, there is no shared language or understanding of what end-of-life care is across health and social care services. Helpfully, Goodman et al (2012) summarise commonly-used terms and their definitions.

What they say is that end-of-life care in care homes is a broad concept; essentially care when death is approaching or support for people with limited life expectancy. The focus in end-of-life care is not simply on the last few weeks/days before death, but when some people think that a resident is in his or her final stage of life. This is a rather circular argument and not helped by the use of images such as 'chapter', or indeed 'stage'. Furthermore, while it may be clear to some people that a resident is approaching end of life, this may not be a shared view or accurate.

What does this mean for care home staff? First, everyone finds it difficult sometimes to say when end of life starts and some people will not use this phrase comfortably. Second, it is not necessarily wrong to have misjudged it; everyone will find some deaths surprising and vice versa. Third, all staff may find different aspects of end-of-life care difficult, but they many also find them hugely rewarding — some of these moments might include holding someone's hand as they die or helping them say goodbye. In a care home, the definition of end of life is likely to be sufficiently broad, to include residents who have moved to the care home for end of life or palliative care, and those who have 'progressive dwindling', a term sometimes used to describe multiple (body and mind) system failure.

Palliative care, with its emphasis on pain management, symptom control and reducing side effects, accounts for a small part of end-of-life care. Palliative care is often talked about as care of the dying, particularly in the last days or weeks before death, where decisions regarding continuing some medical treatments might be made. It is often hard to distinguish between endof-life care and palliative care. 
End-of-life care in a care home encompasses both everyday decisions on how a resident would like to live and die, and thinking about or making arrangements for their future (anticipatory care planning, encompassing preferred place of care and end-of-life plans). Increasing numbers of residents have already made a lasting power of attorney, or have made an advance decision and these must be adhered to under the Mental Capacity Act (2005).

\section{The point of planning}

One key to undertaking end-of-life care is a focus on planning. Planning should consider the resident's personal values, attitudes, beliefs, dignity, relationships and comfort. Ensuring that people die in a dignified, compassionate, peaceful way, and according to their wishes, is important. What seems to be different to end-of-life care in a care home is the presence of other people. This includes other residents, residents' family and friends and also frequently-changing staff. Similarly, what is special about dying in a care home is likely to be influenced by the care home culture and environment, and also by the relationships with a wide range of professionals. These include health-care professionals, but also religious or faith leaders. Everyone may have a different understanding of end of life, and this needs to be considered.

Resident-centred approaches to end-of-life care are encouraged. This may sound like jargon, but it means residents should be at the centre of decisions about their care-including residents with impaired mental capacity — as far as possible. Consideration should also be given to diversity of religion, ethnicity and culture and several of the resources listed below may be helpful in this regard.

\section{Conclusion}

One final observation is that 'care' is the key word when talking about end of life care. Keeping a focus on care is imperative. Staff providing end-of-life care in a care home may be taking on the role of a resident's family member, providing emotional support in addition to other duties. 
Where there are family or friends, these visitors too may need support. It is no surprise that care work is sometimes talked about as 'emotional labour'.

Recognising that staff may feel bereaved or distressed is part of a manager's many jobs.

Managers themselves need support, as they are also likely to have known the resident and their

family, as well as having responsibilities for staff support. 'Who supports the supporters?', is one question were are left to consider when thinking about end-of-life care.

\section{Key Points}

- Providing end-of-life care is likely to be a core part of a care home's business. There are various definitions; therefore, it is helpful to find out what a person means by this term

- It is important for new residents and their family members to know what the care home offers in terms of end-of-life care

- Staff should be helped to understand what is meant by end-of-life care in the care home environment, and why early identification of people approaching end of life may be helpful to all concerned with their care and support

- Care homes should offer bereavement care to staff, as well as residents

- Care home managers themselves may need support when working in a care home providing end-of-life care

\section{Useful Resources}

- End of Life Care Facilitators and Champions Network: http://www2.hull.ac.uk/fass/end-of-life-care-facilitators/members-area.aspx

- National Council for Palliative Care: http://www.ncpc.org.uk/professionals

- Dying Matters: http://www.dyingmatters.org/overview/about-us

- End of Life Care Toolkit:

http://www.southampton.ac.uk/assets/imported/transforms/peripheral- 
block/UsefulDownloads Download/7D8C9310A4C5441E99D7C6B39CB26E88/EoL C-care-home-toolkit.pdf

\section{References}

Office of National Statistics (2012) Mortality Statistics: Deaths Registered in England and Wales. www.ons.gov.uk/ons/rel/vsob1/mortality-statistics--deaths-registered-in-england-and-wales-series- dr-/2012/index.html

National end of life care Intelligence Network (2010) Deaths in Older Adults in England. http://www.endoflifecare-intelligence.org.uk/home

Goodman, C., Froggatt, K. and Mathie, E. (2012) End of Life - research methods review, NIHR School for Social Care Research http://sscr.nihr.ac.uk/PDF/MR/MR12.pdf 